ISSN: 0210-1696

DOI: http://dx.doi.org/10.14201/scero20154645974

\title{
PERFIL Y COMPETENCIAS DEL PROFESIONAL COMO EVALUADOR EN INSTITUCIONES DE ATENCIÓN A PERSONAS CON DISCAPACIDAD INTELECTUAL
}

\section{Profile and Competency on the Professional as Evaluator in Institutions Dedicated to People with Intellectual Disability}

Jesús Miguel Muñoz CANTERo

Departamento de Métodos de Investigación y Diagnóstico en Educación. Facultad de Ciencias de la Educación. Universidad de A Coruña (UDC). Campus de Elviña, s/n. 15071 A Coruña jesus.miguel.munoz@udc.es.

Luisa Losada Puente

Programa de Doctorado en Equidad e Innovación en Educación. Facultad de Ciencias de la Educación. Universidad de A Coruña

Eva María EspiÑ̃iRa Bellón

Área de Métodos de Investigación y Diagnóstico en Educación. Facultad de Ciencias de la Educación. Universidad de A Coruña

Recepción: 13 de mayo de 2015

Fecha de aceptación definitiva: 29 de octubre de 2015

Biblid. [0210-1696 (2015) vol. 46 (4), n. ${ }^{\circ}$ 256, octubre-diciembre; 59-74]

Resumen: La evaluación en el campo de la discapacidad intelectual ha sido relacionada, tradicionalmente, con prácticas basadas en la medición, el control y la sanción, generando cierto rechazo o desinterés por parte de los profesionales debido, entre otros factores, a la ausencia de un perfil profesional definido y formado específicamente para desarrollar esta labor y a las carencias formativas. Este trabajo tiene como objetivo identificar el perfil profesional del evaluador y el tipo de competencias que dominan y aplican las personas evaluadoras en instituciones de atención a personas con discapacidad 
intelectual, y la percepción de estos agentes sobre la necesidad de formación continua. El estudio emplea una metodología cualitativa, de tipo descriptivo, centrada en el análisis documental. Los documentos se recogen por muestreo teórico y se analizan empleando el proceso de codificación teórica. Los resultados indican que la figura del evaluador no se consolida plenamente durante su formación universitaria o profesional, sino que requiere de aprendizaje continuo que le permita actualizarse y comprender mejor la complejidad del ámbito de la discapacidad donde lo primordial es garantizar, por medio de la evaluación, la mejora de los procesos involucrados en la promoción de la calidad de vida de las personas con discapacidad intelectual.

Palabras Clave: evaluadores; discapacidad intelectual; perfil; personal profesional; educación basada en competencias; aprendizaje a lo largo de la vida.

АвsтRAct: Evaluation in the field of intellectual disability has been related, traditionally, to practices based on measurement, control and punishment, generating a rejection or disinterest by professionals due, among other factors, the absence of a professional profile specifically defined and formed to develop this work and training deficiencies of professional evaluators. This paper aims to identify evaluators' professional profile and the type of competency that they master and apply in institutions dedicated to people with intellectual disability, and the perception of these actors on the need for continuous training. The study used a qualitative methodology, descriptive, focus on documents analysis (books, articles, legislation, etc.). Documents are selected by theoretical sampling and are analyzed by a theoretical coding process. The results indicate that the figure of evaluator is not fully consolidated during college or vocational education, but requires continuous learning that allows him to upgrade and better understand the complexity of the field of disability where the primary is to ensure, through evaluation, improvement the processes involved in promoting the quality of life of people with intellectual disabilities.

KEYS WORDs: evaluators; intellectual disability; profile; professional staff; competency based education; lifelong learning.

\section{Introducción}

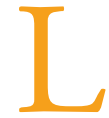

A FIGURA DEL PROFESIONAL EVALUADOR LIGADO A LAS INSTITUCIONES de atención a personas con discapacidad intelectual no deja de ser un tema que ha suscitado ciertas controversias a lo largo del tiempo debido, principalmente, al papel de la propia evaluación como actividad profesional que fue desarrollada inicialmente por personas que se encontraban en posición de poder, autoridad o superioridad respecto de las personas evaluadas y cuyo uso era, fundamentalmente, como un elemento de control, discontinuidad y formalización final de las actividades realizadas. El posterior reconocimiento de su función de mejora y su orientación al perfeccionamiento han permitido ampliar el perfil de personas que se encargan de emitir juicios de valor (Cabra, 2014; Pérez, 2006), estableciéndose una relación directa entre evaluador y sus funciones. 
El papel del evaluador está recogido de diversas maneras en la literatura nacional e internacional, sin que exista un consenso a la hora de definir y/o identificar cuál es realmente su perfil y qué competencias debe tener. La identificación de un perfil general se vuelve más complicada cuando tratamos con profesionales que realizan evaluaciones (de personas, programas, servicios, agentes...) en instituciones que trabajan con personas con discapacidad intelectual. Especialmente, debido al progresivo aumento de la preocupación por evaluar los servicios ofrecidos a este colectivo, valorar la eficiencia de los recursos y la eficacia de los resultados, así como actuar para la mejora continua de la organización, lo cual exige un sistema de organización y gestión interna que dote de formación e información continua a las personas evaluadoras (Schalock y Verdugo, 2007). Nuestro trabajo está orientado a analizar cómo este profesional es percibido en cuanto a las competencias y perfil que debe tener en este marco de intervención.

El profesional evaluador es reconocido, en la mayoría de los casos, como un agente específico y especializado que participa, de forma individual o en colaboración con un equipo de trabajo, en los procesos de intervención, desarrollo y evaluación de los programas institucionales (Castillo y Cabrerizo, 2004). Las características aquí presentadas amplían la diversidad de perfiles derivados de cada ámbito de intervención (educación social, pedagogía, psicopedagogía, terapia ocupacional, etc.) dificultando, de esta forma, la identificación de un perfil claro del evaluador en el campo social y educativo y, más aún, en el de la discapacidad intelectual. A ello se suma el hecho de que en estas instituciones sea habitual la planificación de las actividades, programas y servicios sin definir claramente el perfil de la persona que debe responsabilizarse de la evaluación, basándose en el supuesto de que cualquier persona competente en la identificación de interrogantes, la recogida y el análisis sistemático de los datos puede conducir un proceso evaluativo adecuado (Alkin, 2011; Samperio, 2006).

Frente a esta asunción, diversos autores (Agudo, 2010; McDonald, Bound, Francis y Gonzci, 2009; Scriven, 2007; Stufflebeam y Shinkfield, 2007) reclaman que sólo aquellas personas con una formación y entrenamiento específicos para realizar evaluaciones formales sean identificados como profesionales evaluadores. Estos agentes deberán ser capaces de adoptar un rol diferente en cada momento de su actividad, bien sea como agente externo (ajeno a la institución y al programa y, por tanto, más imparcial en la emisión de juicios), externo al programa pero interno a la institución (menos imparcial y distante pues, aunque su interés no es proteger la imagen del programa, sí lo es la institucional), asesor (apoya y orienta a la persona evaluadora en cuestiones técnicas) o agente interno (influido por el deseo de ofrecer buenos juicios de valor para asegurar la continuidad del programa y de la persona evaluadora en la institución). El tipo de rol que adopte dependerá, por tanto, de su relación con la institución, con el programa evaluado y con las personas implicadas en el proceso.

Para identificar las habilidades, actitudes y aptitudes del evaluador y conocer el tipo de funciones que desempeña en la institución (según el rol adoptado o requerido por ésta) es necesario atender a los conocimientos adquiridos por la persona durante su formación. Al respecto, las investigaciones desarrolladas en los últimos años en materia de formación de evaluadores o referidas a su labor en instituciones sociales y 
educativas evidencian cierta falta de formación a nivel teórico y práctico (Agudo, 2010; Samperio, 2006), y demandan el desarrollo de un marco teórico más sólido y centrado en definir el concepto de evaluación en el desempeño del profesional y en cómo poder mejorar la actividad del evaluador (Tejedor, 2012; Vaillant, 2008). Otros estudios reflexionan sobre la necesidad de dotar a los profesionales de una formación que los capacite y les permita mejorar sus competencias como evaluadores (Agudo, 2010; Cabra, 2014; Leyva, 2012), o acentúan el valor de la formación universitaria para la adquisición de las competencias esenciales para su desarrollo profesional (De Faria, 2010; Martínez y Echeverría, 2009; Martínez, 2008) y la importancia de integrar la evaluación en los procesos de gestión del desempeño profesional (McDonald et al., 2009).

Es responsabilidad de las universidades, en el marco del Espacio Europeo de Educación Superior (EEES), vincular sus titulaciones hacia el sector productivo y organizar los aprendizajes de modo que sean relevantes para la incorporación eficiente de las personas egresadas en el ámbito laboral (Echeverría, 2010; Tejada y Sánchez, 2010). Al adquirir competencias para actuar frente a una situación, el estudiante desarrolla el conocimiento y la capacidad para adecuarse a situaciones específicas, las habilidades para intervenir eficazmente y los valores que le permiten asumir las actitudes acordes con sus principios. Al entender las competencias desde un enfoque holístico, en base a la propuesta de Echeverría (2010), como "Competencias de Acción Profesional” (p. 86), asumimos la importancia de las aptitudes y actitudes, así como los aspectos técnicos y metodológicos, participativos y personales.

Las competencias, una “combinación dinámica de conocimientos, comprensión, habilidades y capacidades, que pueden ser genéricas o específicas” (De Faria, 2010: 19), quedan claramente definidas para los agentes de la intervención socioeducativa (e. g. profesionales de la educación y de la educación social, del trabajo social, de la pedagogía y de la psicología...) (Castillo y Cabrerizo, 2004, 2011; Suárez, 2011); empero, en el caso del agente evaluador, el asunto se vuelve más complejo. Decimos que este agente posee conocimientos y competencias -difíciles de identificar- que comparte con el profesional de la intervención (Agudo, 2010); pero los procesos de preparación específica en evaluación son escasos, pese a la importancia central conferida por las instituciones a la preparación de recursos humanos capacitados (Tejedor, 2012; Ramírez, 2007).

La diversidad de instituciones, procesos y objetos evaluables exige de profesionales con unos conocimientos y competencias específicas en este campo, con una amplia formación teórico-conceptual, metodológico-instrumental y administrativa y de gestión, que les permita desarrollar las competencias necesarias para cada actividad del proceso evaluativo (Agudo, 2010; Ramírez, 2007).

Las actitudes y conocimientos y las destrezas (o competencias) del profesional determinarán las funciones laborales que pueda desempeñar de modo exitoso (Castillo y Cabrerizo, 2011; Martínez, 2008) y su capacidad para mantener la formación y el nivel de cualificación adaptado, en todo momento, a las cambiantes demandas de su profesión (Leyva, 2012; Samperio, 2006). Por ello se justifica la necesidad de analizar las competencias profesionales adquiridas durante la formación universitaria en 
materia de evaluación y, en concreto, en relación con la importancia que les confiere el evaluador que trabaja en una institución de atención a personas con discapacidad. Se pretende en última instancia identificar los perfiles profesionales más demandados por estas instituciones.

\section{Objetivos}

Nuestro objetivo es identificar los perfiles dominantes y las competencias de las que los evaluadores deberían disponer en su desempeño profesional en instituciones socioeducativas de atención a personas con discapacidad, así como identificar los aspectos claves en los que debería basarse la formación continua a lo largo de la vida.

\section{Método}

Partimos de una metodología cualitativa, basada en la investigación documental en tanto es un procedimiento científico extenso, universalizado y sistemático de indagación, recolección, organización, reflexión, análisis e interpretación de información acerca de un tema concreto para la construcción del conocimiento (Atkinson y Coffey, 2011; Bernete, 2013; Ruiz, 2012). El estudio de la cultura material es de gran importancia para el investigador cualitativo que desea explorar las múltiples voces que ofrecen interpretaciones diferentes e interconectadas, permitiendo comparar documentos y atender a cuestiones que no hubiera sido posible comprender y explicar extensivamente desde una metodología puramente cuantitativa (Andreu y Labrador, 2011; Bernete, 2013).

Nuestra pretensión es situarnos en un primer nivel descriptivo que nos facilite el descubrimiento de los componentes básicos del fenómeno de la evaluación y del agente evaluador y, posteriormente, alcanzar un mayor nivel de abstracción al interpretar y dar una explicación al fenómeno de estudio (Andreu y Labrador, 2011; Flick, 2007; Glaser y Strauss, 2012; Ruiz, 2012).

El estudio se estructura, tomando como referentes las investigaciones documentales de Bernete (2013) y Monroy y Hernández (2014) en 3 fases, las cuales se desarrollan en el marco de un diseño circular o emergente, donde las prácticas que llevamos a cabo son de carácter recurrente; esto es, el estudio es reformulado y reconducido constantemente en cada fase del proceso investigador:

Fase 1. Trabajo previo a la obtención de datos: iniciamos la investigación con la formulación del problema y de los objetivos del estudio, así como la identificación de los contextos de trabajo que suscitan interés para el estudio (en nuestro caso, las principales áreas socioeducativas directamente vinculadas con la discapacidad y la evaluación en el campo de la discapacidad). A partir de ello, se definieron los criterios de selección de los datos; concretamente, documentos que planteasen enfoques diversos para la evaluación en el terreno de la discapacidad y que identificasen, 
directa o indirectamente, la formación y conocimientos del evaluador y su labor en el campo de acción.

Fase 2. Extracción de datos o "trabajo de campo": se procedió a la recogida de material escrito (fuentes primarias y secundarias) a través del muestreo teórico (Glaser y Strauss, 2012), basado en la codificación y análisis sistemático de cada documento con lo que poder definir las características de los siguientes datos a recolectar.

Revisamos la literatura existente realizando varias búsquedas en bases de datos españolas (DIALNET, CSIC-ISOC) y en el Repositorio Institucional de la Universidad de A Coruña. En primer lugar, introdujimos cuatro palabras clave o descriptores (evaluación, evaluador, discapacidad o atención a la diversidad), obteniendo más de 200 documentos que fueron filtrados por título y resumen. Descartamos aquellos artículos que contextualizaban realidades diferentes a la socioeducativa, al colectivo de personas con discapacidad o que no hiciesen mención a la figura del evaluador socioeducativo. También se descartaron las publicaciones anteriores al año 1995, priorizando las más actuales, puesto que el interés era conocer el estado actual de la cuestión. Una vez analizados los textos extraídos (un total de 14), realizamos una nueva búsqueda afinando los descriptores (evaluación democrática, desempeño profesional, competencias, funciones, perfiles, roles) y manteniendo conceptos clave como "evaluador" y "discapacidad". Obtuvimos 52 documentos que fueron filtrados utilizando el criterio anteriormente mencionado hasta quedarnos con nueve nuevos documentos para análisis. Finalmente, repetimos el procedimiento, introduciendo los descriptores "calidad de vida”, "evaluación de programas”, "resultados personales” y "discapacidad intelectual”, extrayendo cinco nuevos documentos de interés. El resultado final fue un total de 28 documentos clave para la construcción de nuestra teoría.

Fase 3. Explotación de los datos: partiendo de los presupuestos de la Teoría Fundamentada, el análisis e interpretación de la información se desarrolló en paralelo a la recogida de datos, de modo que la información extraída de cada documento orientase el camino para la búsqueda de nueva información. Así, el análisis se desarrolló mediante el proceso de codificación teórica, el cual se divide en tres actividades: reducción y simplificación de la información; codificación y categorización de la información, e interpretación de las categorías. La reducción o simplificación de la información en unidades nos permite convertir la información en abarcable y manejable y segmentar los elementos más relevantes y significativos para el estudio. Cada documento textual constituyó una unidad de análisis. Dichas unidades fueron diferenciadas en unidades temáticas, en función de si el tema central del texto era la discapacidad, la evaluación o el evaluador. A continuación, se inició un proceso en tres fases: codificación abierta, codificación axial y codificación selectiva (Flick, 2007, 2014; Taylor y Bodgan, 2005).

En la fase de codificación abierta, categorizamos y codificamos los datos para mejorar la comprensión de los textos. Este proceso resultó en un elevado número de códigos con los que creamos las categorías iniciales o subcategorías. Así, por ejemplo, aquellos códigos referidos a las actividades del evaluador, la aplicación de competencias, las competencias de acción profesional, las competencias en evaluación, el tipo de 
competencias, el tipo de roles, las dificultades en la evaluación, la complementariedad de funciones o la relación entre perfil-competencias, roles y funciones, fueron categorizados en cinco subcategorías referidas a las Competencias, al Perfil, al Rol, a las Funciones y al Desempeño Profesional del Evaluador.

En la fase de codificación axial dotamos de un sentido de causa-consecuencia a las relaciones entre códigos y subcategorías. Posteriormente, elevamos y saturamos las subcategorías hasta extraer las categorías centrales de la investigación. Por ejemplo, las subcategorías “Competencias”, "Perfil”, "Rol” y "Funciones” se elevaron a la categoría “Características del evaluador”; o la subcategoría "Desempeño Profesional”, junto con el "Tipo de evaluación en el ámbito de la discapacidad”, o la "Mejora de la calidad de vida en la discapacidad" se integraron en la categoría "Evaluación en instituciones de atención a personas con discapacidad intelectual”; y, por último, se creo la categoría "Valor del concepto de evaluación" a partir de las subcategorías "Valoración del proceso de evaluación” y "Estimación del trabajo del evaluador".

Finalmente, en la fase de codificación selectiva finalizamos la recogida de datos al considerar que cualquier información adicional podría ser contraproducente para la generación de nuestra teoría; esto es, alcanzamos la saturación teórica (Glaser y Strauss, 2012). En esta fase logramos un nivel mayor de abstracción, al obtener un concepto central que regula todo el proceso investigador: la figura de la persona evaluadora, en base al que formulamos el relato o interpretación de la investigación.

\section{Resultados}

El análisis documental nos ha permitido la extracción de tres categorías principales que conforman la figura del evaluador, y que presentamos continuación:

\section{El valor del concepto de evaluación}

La evaluación se sitúa como la "disciplina más fundamental de la sociedad" (Stufflebeam y Shinkfield, 2007: 4), cuya utilidad permite el desarrollo racional de las acciones humanas en cualquier ámbito social (Samperio, 2006). Por ello, analizar el valor de este concepto implica referirse a dos acepciones: la primera, referida a la valoración en general que se hace del proceso de evaluación, entendido como un elemento esencial de la actividad humana, y la segunda, a la estimación que el profesional evaluador hace hacia esta actividad, es decir, a la utilidad que le confiere a su práctica (véase Figura 1), y que se relaciona con la capacidad de este agente para valorar y autoevaluar su trabajo y atender al modo en que podría mejorarlo.

La evaluación es parte imprescindible de las actividades cotidianas y de la actividad profesional pues facilita la comprensión, valoración y apoyo a la mejora del aspecto evaluado. Es una necesidad para todos los profesionales del sector socioeducativo ya que permite tomar decisiones sobre asuntos vinculados a su práctica que afectan positiva o negativamente al desarrollo del proyecto de intervención (Castillo y Cabrerizo, 
2011). Su finalidad, por tanto, no es sólo informar sobre el resultado del programa, proyecto o servicio, sino dotar de datos relevantes sobre el modo de percibir la validez y el valor del evaluador en relación con la persona evaluada (Zufiaurre y Albertín, 2006).

\section{FIGURA 1. Mapa conceptual: “El valor del concepto de evaluación”}

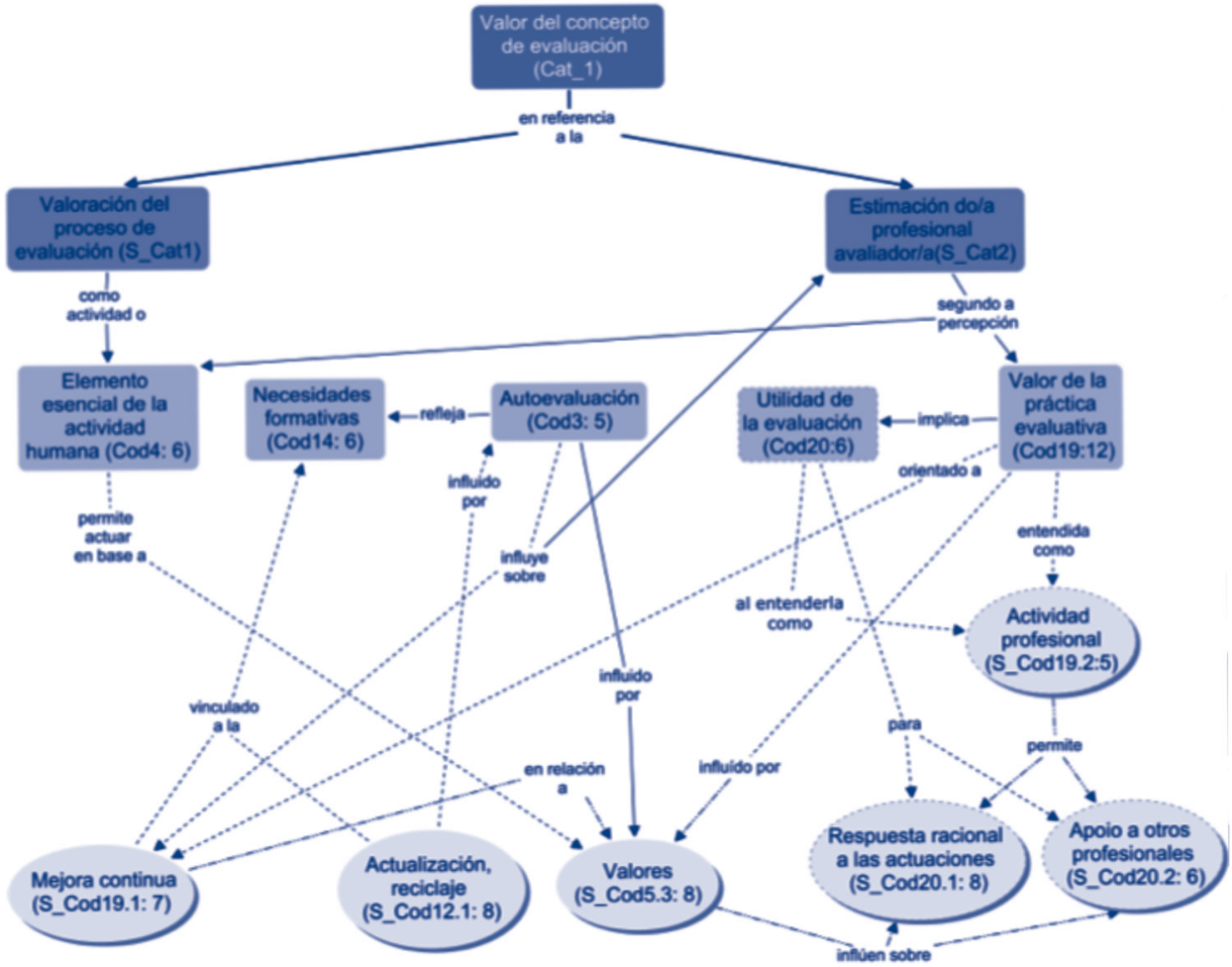

Se presenta, con esta figura, el resultado del análisis por medio de codificación teórica, donde se dedujo la importancia de ofrecer una definición clara sobre el significado del concepto de evaluación, atendiendo fundamentalmente a la valoración ofrecida a este proceso y la estimación que los profesionales evaluadores hacen de la misma.

Fuente: Elaboración propia, a partir del programa CMapsTools.

El valor que la persona evaluador le confiere a su actividad, junto con sus valores y sus juicios, podrá tener repercusión sobre el concepto y la utilidad que se le da a la evaluación. Esto es, una valoración positiva de las prácticas de evaluación puede ser un aliciente para el apoyo a otras disciplinas, profesionales, stakebolders (personas evaluadas), etc. así como un elemento que motive la autoevaluación orientada a la actualización y la mejora, con el fin de generar un beneficio para el conjunto de agentes que integran la organización (profesionales evaluadores, usuarios, familias, etc.).

(C) Ediciones Universidad de Salamanca

Siglo Cero, vol. 46 (4), n. ${ }^{\circ} 256,2015$, octubre-diciembre, pp. 59-74

$$
-66-
$$


La creciente importancia de la evaluación en la sociedad y en la educación está propiciando el surgimiento de nuevos desafíos para las prácticas evaluativas tradicionales, que repercuten en la demanda de nuevos modos de entender y aplicar la evaluación, y de respuestas innovadoras por parte de los agentes profesionales y las instituciones (Castillo y Cabrerizo, 2011; McDonald et al., 2009). Tales cambios afectan, de modo especial, al sector de la discapacidad intelectual, como resultado de la introducción de un nuevo concepto entre las prácticas profesionales, la calidad de vida (Schalock, 2003; Schalock y Verdugo, 2007; Verdugo, 2009).

La mejora de la calidad de vida de las personas con discapacidad exige un compromiso de las instituciones y profesionales por adecuarse y centrarse en las necesidades de los usuarios y en la planificación individual respecto a las metas y las técnicas que afectan a sus vidas. Justifica esto la demanda de profesionales que entiendan la evaluación como el resultado de la interacción de numerosas variables que contribuyen a aportar una visión más global y equilibrada del potencial de las personas con discapacidad y que dedique un espacio para la reflexión sobre su praxis profesional (Castillo y Cabrerizo, 2011; Suárez Riveiro, 2011); esto es, un agente interesado por analizar y reflexionar sobre su propio quehacer, que le permita su crecimiento personal y profesional, así como el desarrollo de un proceso de mejora constante, al analizar su competencia para llevar a cabo las actividades exigidas en su contexto de trabajo.

\section{Las características del profesional evaluador}

La evaluación es una actividad accesible a cualquier persona que intenta comprender qué aspectos que facilitan o dificultan sus actuaciones y cómo poder mejorarlas; no obstante, lo que distingue al evaluador de otros agentes con los que comparte espacios de trabajo es su experiencia, su conocimiento profundo de la materia, su modo de pensar diferente en la toma de decisiones diarias y su capacidad para identificar interrogantes, recoger y analizar sistemáticamente los datos (Alkin, 2011; McDonald et al., 2009; Mertens y Wilson, 2012). Se trata de un profesional formado e informado sobre la actividad que desarrolla, que presta especial atención a su contexto específico de trabajo y a las características de los usuarios con los que interviene, y a su rol en la institución (véase Figura 2).

Para el desarrollo de sus funciones (esto es, análisis de la realidad, diseño y diseño de la evaluación, comunicación y difusión de los resultados, implicación y cooperación con otros profesionales, transmisión de conocimientos y respeto a los principios éticos y de responsabilidad), el evaluador precisa de unos conocimientos, habilidades y destrezas; esto es, la persona tiene los conocimientos teóricos apropiados, pero también la capacidad para aplicarlos a las condiciones y al contexto específico, además de una serie de valores acordes con sus principios de actuación. Este conjunto de saberes, denominado Competencia de Acción Profesional (CAP), determinan la capacidad del profesional de trasladar a la práctica su competencia técnica (saber), metodológica (saber hacer), participativa (saber estar) y personal 
(saber ser), de un modo consecuente con las exigencias del lugar de trabajo y de las personas implicadas.

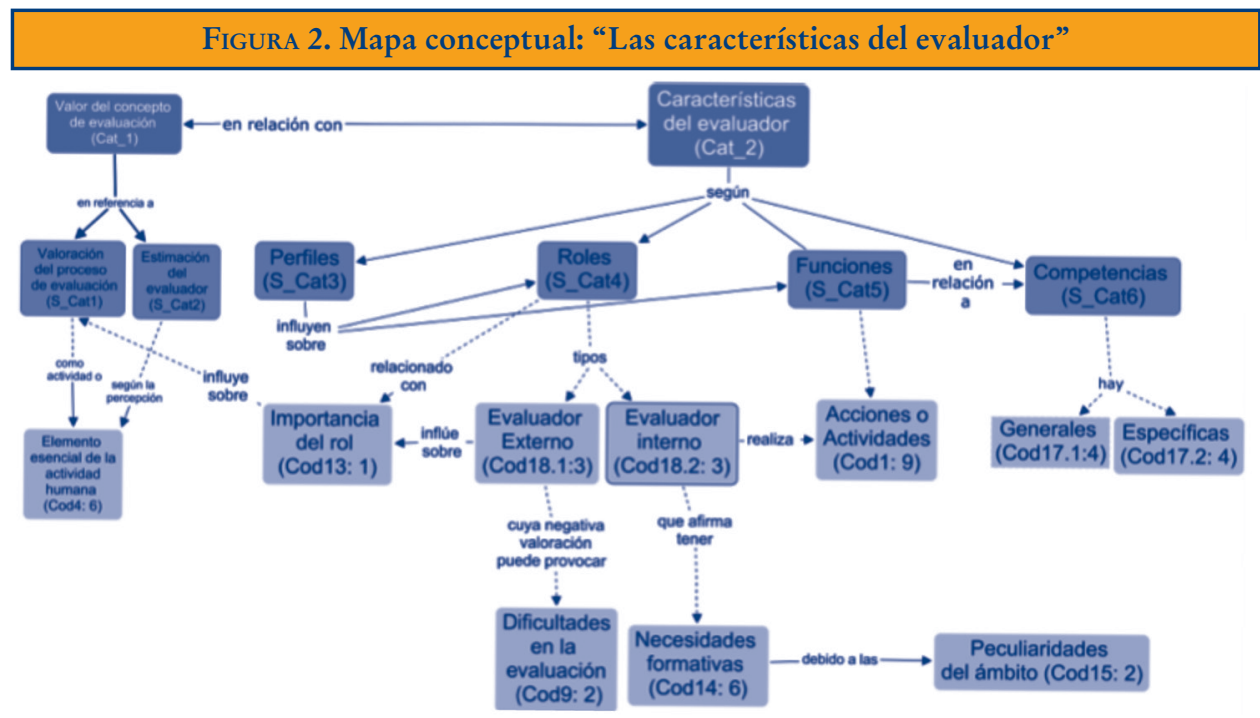

En esta figura se presentan las principales características del evaluador, en base a la producción científica analizada, así como en relación con la valoración y percepción de los profesionales acerca del proceso evaluativo.

Fuente: Elaboración propia, a partir del programa CMapsTools.

En el caso del evaluador, las fuentes consultadas (Leyva, 2012; McDonald et al., 2009; Samperio, 2006; Stufflebeam y Shinkield, 2007) nos permiten explicitar aquellas competencias que deberían ser adquiridas por cualquier agente que, trabajando en el campo socioeducativo, se dedique a realizar evaluaciones de actividades, programas, proyectos y servicios en instituciones. Éstas son: (a) conocer los fundamentos teóricos de la evaluación social y educativa; (b) ser capaz de comprender y criticar informes de investigación y evaluación social y educativa; (c) ser flexible para proponer alternativas ante diversas situaciones; (d) conocer la metodología general de evaluación social y educativa; (e) conocer los procedimientos e instrumentos de recogida y análisis de información; (f) ser capaz de realizar análisis sistemáticos de la información; (g) ser capaz de adoptar las diversas perspectivas evaluativas según las necesidades del grupo/persona; (h) ser capaz de emplear la comunicación oral y escrita de modo empático y responsable a los diversos implicados, en la transmisión de los resultados de diagnóstico, seguimiento y evaluación final; (i) actuar con transparencia en la difusión de la metodología empleada en el proceso evaluativo; (j) ser capaz de combinar la teoría y las habilidades técnicas en la práctica de la evaluación social y educativa; $(\mathrm{k})$ interesarse por la actualización permanente en el campo de la evaluación social y educativa; (l) interesarse por la investigación y por los procesos de metaevaluación; $(\mathrm{m})$ conocer y comportarse de acuerdo con las responsabilidades y 
normas éticas del agente evaluador; y (n) ser capaz de resolver posibles conflictos entre las diversas audiencias implicadas en un proceso evaluativo.

Estas quince competencias atienden a los cuatro dominios de la CAP: teóricoconceptual (saber), metodológico-instrumental (saber hacer), social y participativo (saber estar) e integrativo-adaptativo (saber ser). También destacamos otros aspectos como la credibilidad, la motivación, la orientación y buenos hábitos en el trabajo, la integridad, el espíritu colaborativo, la asignación clara y relevante, la disposición de los medios y recursos necesarios para el trabajo y la formación continua, la adecuada compensación, la retroalimentación y la supervisión receptiva y centrada en el apoyo (Stufflebeam y Shinkfield, 2007: 121).

Con ello, podemos afirmar que tanto las actitudes, como los conocimientos y las destrezas del evaluador determinarán las funciones que pueda desarrollar de un modo exitoso, así como su capacidad de mantenerse en continua formación, especialización y el nivel de cualificación adaptado a las cambiantes demandas sociopolíticas y económicas de su profesión y de los contextos de empleo (Castillo y Cabrerizo, 2011; McDonald et al., 2009; Samperio, 2006; Stufflebeam y Shinkfield, 2007; Zufiaurre y Albertín, 2006).

\section{La evaluación en instituciones de atención a personas con discapacidad intelectual}

El discurso actual en torno a la evaluación en el ámbito de la discapacidad está dando un especial valor al seguimiento de todas las actuaciones de los profesionales, como procedimientos que permiten analizar y gestionar el transcurso de las prácticas de intervención con las personas con discapacidad intelectual atendiendo a la mejora y a la calidad de éstas (Calero, Fontcuberta, García, Ballesteros y De Wispelaere, 2013).

La evaluación, como proceso reflexivo que involucra a todas las personas que participan en el funcionamiento de la institución (trabajadores/as, usuario/as, familias, etc.), debería “proporcionar información suficiente para poder llegar a asegurar la calidad de un servicio, y también para mejorarlo” (Zufiaurre y Albertín, 2006: 76).

El análisis de la figura del evaluador en el campo de la discapacidad intelectual nos revela la heterogeneidad que enmarca a este contexto, reflejándose en la selección de teorías y modelos de evaluación, así como de los tipos de evaluación más adecuados a cada contexto y circunstancia. De ahí la importancia de identificar a los actores sociales y educativos que trabajarán con estas personas y analizar su postura hacia las funciones que le otorgan como evaluador, pues la actitud del profesional podrá repercutir en su quehacer diario, en la valoración de su actividad y en sus expectativas de superación personal (Calero et al., 2013; House, 1997).

$\mathrm{La}$ actitud y la responsabilidad del profesional ante su desempeño profesional serán de suma importancia, por ser valores inherentes a la persona, no aprendidos durante la formación en competencias, y que repercutirán enormemente sobre su modo de desarrollar la evaluación en instituciones de atención a personas con discapacidad intelectual (véase Figura 3).

Pero, además, debemos tener en cuenta que la comprensión de la evaluación por parte de cada agente estará influida por sus valores y actitud cuya influencia se extiende al desarrollo de su actividad. El progresivo enfoque de la evaluación hacia la 
consecución de mayores estándares de rendimiento puede estar provocando una actitud de rechazo de los profesionales, al entender la evaluación como medida de control y sanción, de rendición de cuentas y como elemento para la satisfacción de intereses particulares, en vez de situarse como un proceso vinculado a la mejora y a la calidad de las acciones profesionales.

Las dificultades para comprender su finalidad, debido a la ausencia de unos objetivos claros y definidos, así como de unas pautas que orienten a los profesionales en el desarrollo de su labor, no solo repercuten sobre el desempeño del profesional, sino que afectan, en gran medida, a las actuales pretensiones de mejorar la calidad de vida de las personas con discapacidad intelectual, obligando a cuestionarse el papel que juegan las personas con discapacidad como implicadas o excluidas en los procesos de evaluación; esto es, el modo en que las organizaciones sociales trabajan con las personas con discapacidad y no para ellas.

\section{FIGURA 3. Mapa conceptual: “La evaluación en instituciones de atención} a personas con discapacidad intelectual"

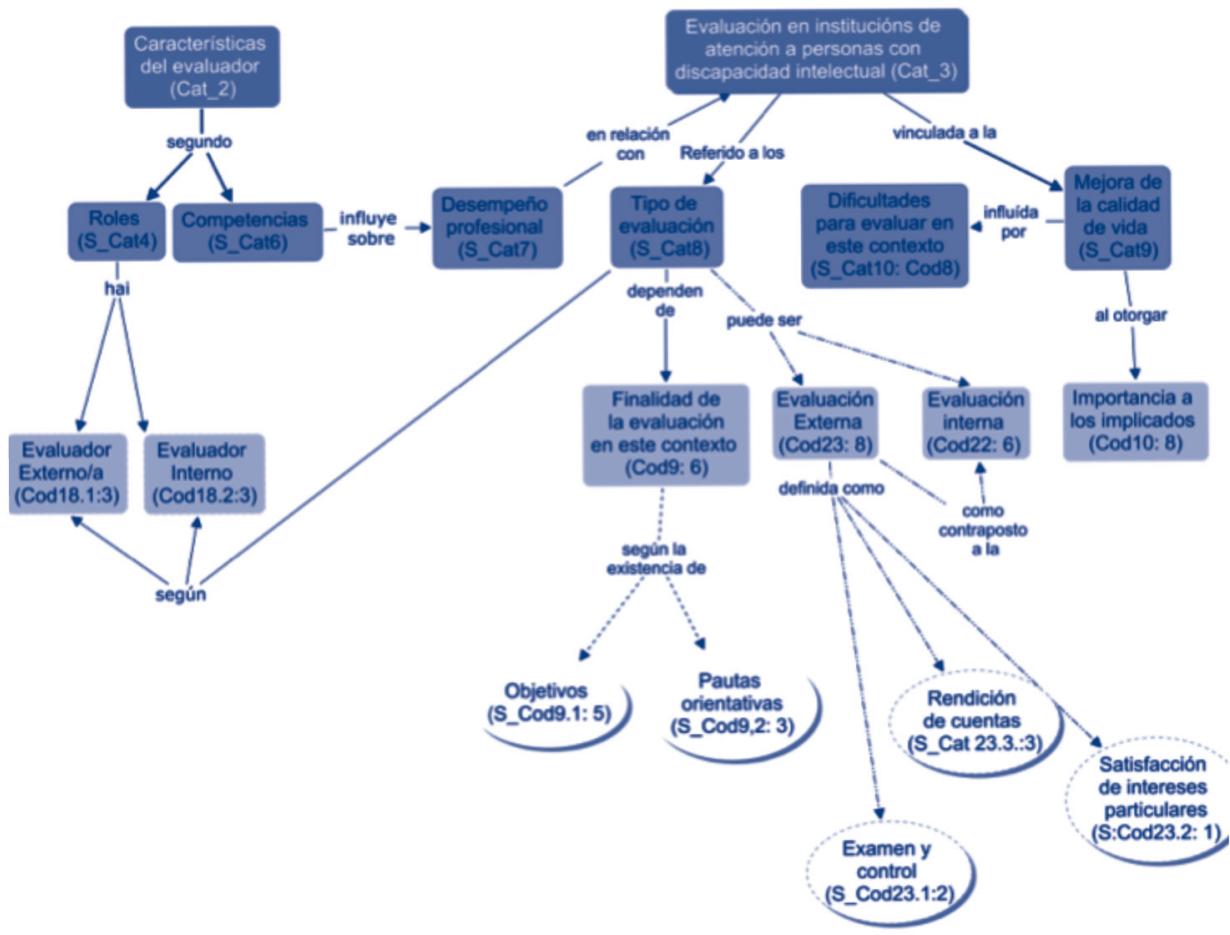

Esta representación gráfica ofrece información referente a los procesos evaluativos en instituciones que atienden a personas con discapacidad intelectual (desempeño del evaluador, tipos de evaluación, dificultades relativas a la evaluación en este ámbito, etc.), tomando como referente el rol del evaluador interno a la institución.

Fuente: Elaboración propia, a partir del programa CMapsTools. 


\section{Conclusiones}

Este trabajo contribuye a dar respuesta a una serie de interrogantes en torno a la figura de la persona evaluadora en instituciones de atención a personas con discapacidad. Al atender a la evaluación como actividad profesional e indagar sobre el perfil predominante y las competencias del evaluador en instituciones que trabajan con personas con discapacidad, podemos caracterizarlo como un profesional con un rol de agente interno a la institución en la que trabaja y con una formación, preferentemente, en una de las disciplinas del ámbito de las Ciencias Sociales y Educativas.

La evaluación en este campo es un aspecto de creciente relevancia, más aún en lo referente a la valoración de los servicios y los apoyos que se ofrecen a las personas con discapacidad desde instituciones que promueven un modelo social de discapacidad centrado en la mejora de la calidad de vida de las personas con discapacidad. En las instituciones con las que trabajamos, nos encontramos con profesionales cuyas prácticas comienzan a acercarse a las propias de un modelo social de la discapacidad, como son el énfasis en la interdisciplinariedad y el trabajo conjunto, los intentos por dar voz a las personas con discapacidad, implicarlas en las acciones profesionales y la búsqueda de mecanismos que permitan trabajar con ellos y no para ellos.

El empleo de un modelo que tenga en cuenta los diversos puntos de vista, que analice objetiva y subjetivamente el funcionamiento de los centros y de los servicios hacia la promoción de los cambios, contribuye a la creación de una cultura de evaluación basada en la reflexión compartida por parte de las personas evaluadoras en torno a sus prácticas, a los resultados y a la promoción de la mejora en la atención a las personas con discapacidad y a sus familias.

Sin embargo, la perspectiva de la persona evaluadora ha sido, hasta el momento, poco estudiada y estimada en este ámbito. Los profesionales que intervienen y evalúan en estas instituciones demandan ser parte activa de su equipo de trabajo, protagonistas de la reflexión y de las propuestas de mejora. Para hacerlo, se sirven de sus conocimientos en materia de evaluación, sobre todo de aquellos que se orientan a la adecuación del saber a la práctica, como son la capacidad para analizar de modo sistemático la información; para adoptar enfoques diversos según las necesidades del grupo o persona; la capacidad para comunicarse de forma empática y responsable con las personas implicadas en la transmisión de los resultados de evaluación, y la actuación con transparencia en la difusión de la metodología empleada en el proceso evaluativo.

El valor de este estudio reside en la necesidad de ampliar el conocimiento en torno a las dificultades y retos a los que se enfrentan los profesionales, facilitando la apertura de canales para la mejora de la formación y las prácticas que contribuyan al desarrollo de evaluaciones más comprensivas, útiles, cooperativas y transformadoras. Nuestro análisis nos permite recoger información exhaustiva sobre la figura del evaluador, con la que poder diseñar instrumentos más precisos para su estudio en profundidad. Se propone, por ello, una definición operativa del agente evaluador, que sirva de marco en el diseño de un cuestionario que recoja información sobre el perfil, formación en competencias y desempeño profesional del agente evaluador en instituciones de atención a personas con discapacidad intelectual (véase Tabla 1). 
Concluimos evidenciado la importancia del estudio en profundidad de la formación profesional en evaluación en función de las competencias de cada titulación, así como sobre los enfoques ofrecidos y adoptados para su aplicación, pues ésta podría acercarnos algo más a la comprensión del modelo actual sobre el que se asientan las prácticas y las intervenciones con las personas con discapacidad intelectual.

\begin{tabular}{|c|c|c|}
\hline \multicolumn{3}{|c|}{ Tabla 1. Definición operativa de la figura del evaluador } \\
\hline Categorías & Dimensiones & Indicadores \\
\hline Evaluación & $\begin{array}{l}\text { Utilidad de la } \\
\text { evaluación }\end{array}$ & $\begin{array}{l}\text { - Utilidad de la evaluación inicial, procesual y } \\
\text { final } \\
\text { - Utilidad de la evaluación como apoyo al } \\
\text { desarrollo de las tareas profesionales }\end{array}$ \\
\hline \multirow{3}{*}{$\begin{array}{l}\text { Características del } \\
\text { evaluador }\end{array}$} & \multirow{3}{*}{$\begin{array}{c}\text { Formación } \\
\text { adquirida en materia } \\
\text { de evaluación }\end{array}$} & $\begin{array}{l}\text { Competencias } \\
\text { - Teórico-conceptual (Saber) } \\
\text { - Metodológico-instrumental (Saber hacer) } \\
\text { - Social y participativa (Saber estar) } \\
\text { - Integrativa-adaptativa o personal (Saber ser) }\end{array}$ \\
\hline & & $\begin{array}{l}\text { Perfil dominante } \\
- \text { Titulación de mayor nivel (formación } \\
\text { profesional, licenciatura, diplomatura, grado) } \\
\text { - Formación de posgrado en evaluación } \\
\text { (máster, doctorado) }\end{array}$ \\
\hline & & $\begin{array}{l}\text { Necesidades formativas } \\
- \text { En competencias teórico-conceptuales (Saber) } \\
- \text { En competencias metodológico- } \\
\text { instrumentales (Saber hacer) } \\
- \text { En competencias sociales y participativas } \\
\text { (Saber estar) } \\
- \text { En competencias integrativo-adaptativas o } \\
\text { personales (Saber ser) }\end{array}$ \\
\hline $\begin{array}{l}\text { Evaluación en } \\
\text { el ámbito de la } \\
\text { discapacidad } \\
\text { intelectual }\end{array}$ & $\begin{array}{l}\text { Desempeño } \\
\text { profesional }\end{array}$ & $\begin{array}{l}\text { - Aplicación de las competencias en materia de } \\
\text { evaluación } \\
\text { - Competencias específicas en evaluación en la } \\
\text { discapacidad } \\
\text { - Complementariedad de funciones de } \\
\text { intervención y evaluación } \\
\text { - Tipo de funciones en materia de evaluación y } \\
\text { específicas del campo de la discapacidad } \\
\text { - Dificultades experimentadas }\end{array}$ \\
\hline \multicolumn{3}{|c|}{$\begin{array}{l}\text { Se concluye esta investigación planteando el diseño de un cuestionario con el que } \\
\text { poder indagar con mayor profundidad en esta figura. La información contenida en dicho } \\
\text { cuestionario responde a los planteamientos teóricos analizados a lo largo del estudio. }\end{array}$} \\
\hline
\end{tabular}

Fuente: Elaboración propia. 


\section{Referencias bibliográficas}

Agudo, M. C. (2010). La formación del evaluador educacional. Academia Nacional de Educación. Recuperado de http://www.educ.ar/dinamico/UnidadHtml_get_2699e6d27a08-11e1-83d9-ed15e3c494af/Formacion_evaluador_Agudo.pdf.

Alkin, M. C. (2011). Evaluation essentials: from A to Z. New York, EE. UU.: The Guilford Press.

Andreu, M. A. y Labrador, M. J. (2011). Formación del profesorado en metodologías y evaluación. Análisis cualitativo. Revista de Investigación en Educación, 2 (9), 136-245. Recuperado de http://hdl.handle.net/10251/29188.

Atrinson, P. y Coffey, A. (2011). Analysing Documentary Realities. En D. Silverman (Ed.), Qualitative research (pp. 77-92) (3.a ed.). Londres, Reino Unido: Sage.

Bernete, F. (2013). Análisis de contenido. En A. L. Marín y A. Noboa (Coords.), Conocer lo social: estrategias y técnicas de construcción y análisis de datos (pp. 193-225). Madrid: Fragua.

Cabra, F. (2014). Evaluación y formación para la ciudadanía: una relación necesaria. Revista Iberoamericana de Educación, 64, 177-193. Recuperado de http://www.rieoei.org/rie64a11.pdf.

Calero, J. (Dir.), Fontcuberta, X. (Coord.), García, A., Ballesteros, A. y De Wispelaere, J. (2013). Guía para la evaluación de programas y políticas públicas de discapacidad. Madrid: CERMI.

Castillo, S. y Cabrerizo, J. (2004). Prácticas de evaluación educativa: materiales e instrumentos. Madrid: Pearson Educación.

Castillo, S. y CABrerizo, J. (2011). Evaluación de la intervención socioeducativa: antes, ámbitos y proyectos. Madrid: UNED.

De Faria, E. (2010). La importancia de las competencias en la educación superior. Cuadernos de Investigación y Formación en Educación Matemática, 5 (6), 13-37. Recuperado de http:// revistas.ucr.ac.cr/index.php/cifem/article/download/6921/6607.

Echeverría, B. (2010). Competencias y cualificaciones de acción. En B. Echeverría (Coord.),

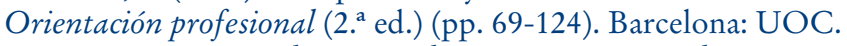

Flick, U. (2007). Introducción a la investigación cualitativa (trad. A. Gallardo) (4. ${ }^{a}$ ed.). Madrid: Morata.

Flick, U. (2014). An introduction to qualitative research (5. ${ }^{a}$ ed.). London: Sage.

House, E. (1997). Evaluación, ética y poder (trad. A. Gallardo) (2. ${ }^{a}$ ed). Madrid: Morata. Madrid: Narcea.

LeYva, Y. E. (noviembre, 2012). Concepto actual de evaluación educativa y las competencias del evaluador. Trabajo presentado en el I Foro Iberoamericano de Evaluación Educativa. Baja California, México.

MartíneZ, J. (2008). La opinión de los profesores universitarios. Trabajo presentado en el Seminario del Instituto de Ciencias de la Educación. Universidad de Zaragoza, Zaragoza.

Martínez, P. y Echeverría, B. (2009). Formación basada en competencias. Revista de Investigación Educativa, 27 (1), 125-147. Recuperado de http://www.redalyc.org/articulo. oa? id $=283322804008$.

McDonald, R., Bous, D., Francis, J. y Gonczi, A. (2009). Nuevas perspectivas sobre la evaluación. Sección para la Educación Técnica y Profesional, 149. Recuperado de http://www. oei.es/etp/nuevas_perspectivas_evaluacion.pdf.

Mertens, D. M. y Wilson, A. T. (2012). Program evaluation theory and practice. A comprebensive guide. Nueva York, EE. UU.: The Guildford Press.

Monroy, F. y Hernández, F. (2014). Factores que influyen en los enfoques de aprendizaje universitario. Una revisión sistemática. Educación XXI, 17 (2), 105-124. doi.: 10.5944/ educxx1.17.2.11481 
PERFIL Y COMPETENCIAS DEL PROFESIONAL COMO EVALUADOR EN INSTITUCIONES DE ATENCIÓN... JESÚS MIGUEL MUÑOZ CANTERO, LUISA LOSADA PUENTE Y EVA MARÍA ESPIÑEIRA BELLÓN

Pérez, R. (2006). Evaluación de programas educativos. Madrid: La Muralla.

RAMíREZ, M. S. (2007). La formación de evaluadores institucionales en modelos de educación a distancia: resultados preliminares de un estudio complementario. Trabajo presentado en el IX Congreso Nacional de Investigación Educativa. Mérida, México.

Ruiz, J. L. (2012). Análisis de contenido. En J. L. RuIz (Ed.), Metodología de la investigación cualitativa (pp. 191-214) (vol. 15) (5. ${ }^{a}$ ed.). Bilbao: Deusto.

Samperio, L. M. (2006). Apuntes acerca de la evaluación y la certificación de la competencia laboral. En C. BARrón (Coord.), Proyectos educativos innovadores. Construcción y debate (pp. 52-81). Coyoacán, México: Universidad Nacional Autónoma de México (UNAM).

Schalock, R. L. (2003). Medida de los resultados y gestión de los resultados: el papel de la evaluación basada en los resultados. Revista Española sobre Discapacidad Intelectual, 34 (2), 56-67. Recuperado de http://riberdis.cedd.net/handle/11181/3063?rd=0031937174951658.

Schalock, R. L. y Verdugo, M. Á. (2007). El concepto de calidad de vida en los servicios y apoyos para las personas con discapacidad intelectual. Revista Española sobre Discapacidad Intelectual, 38 (4), 21-36. Recuperado de http://riberdis.cedd.net/handle/11181/3169? $\mathrm{rd}=0031588695976418$.

Scriven, M. (2007). The logic of evaluation. En H. V. Hansen (Ed.), Dissensus and the Search for Common Ground (pp. 1-16). Windsor, Canadá: OSSA.

Stufflebeam, D. L. y Shinkfield, A. J. (2007). Evaluation theory, models, and applications. San Francisco, EE. UU.: Jossey-Bass.

SuÁrez, J. M. (2011). Discapacidad y contextos de intervención. Madrid: Sanz y Torres.

TAYlor, S. J. y Bogdan, R. (2005). Introducción a los métodos cualitativos de investigación (trad. J. Piatgorski) (5. ${ }^{a}$ ed.). Madrid: Morata.

Tejada, R. y SÁnchez del Toro, P. (2010). Estrategias de intervención para la formación de competencias profesionales en la educación superior. Revista Pedagogía Universitaria, 15 (5), 39-53. Recuperado de http://cvi.mes.edu.cu/peduniv/index.php/peduniv/article/ view/554/553.

Tejedor, F. J. (2012). Evaluación del desempeño docente. Revista Iberoamericana de Evaluación Educativa, 15 (1e), 318-327. Recuperado de http://rinace.net/riee/numeros/ vol5-num1_e/art24_htm.htm.

Vaillant, D. (2008). Algunos marcos referenciales para la evaluación del desempeño docente en América Latina. Revista Iberoamericana de Evaluación Educativa, 1 (2), 7-22. Recuperado de http://fudespa-org/extras/evaluacion_del_desempeno_docente.pdf.

Verdugo, M. Á. (2009). Calidad de vida, I+D+I y políticas sociales. Siglo Cero. Revista Española sobre Discapacidad Intelectual, 40 (2), 5-21. Recuperado de sid.usal.es/artículos/40-8-229/calidad-de-vida-i-d-i-y-politicas-sociales.aspx.

Zufiaurre, B. y Albertín, A. (2006). Evaluación democrática y proyectos cooperativos. El lugar de una evaluación de calidad en una cultura escolar democrática. Navarra: Universidad Pública de Navarra.

(C) Ediciones Universidad de Salamanca

Siglo Cero, vol. 46 (4), n. ${ }^{\circ}$ 256, 2015, octubre-diciembre, pp. 59-74 